\title{
Low-repetition-rate Integrated Electro-optic Frequency Comb Sources
}

\author{
Amirhassan Shams-Ansari ${ }^{1}$, Christian Reimer ${ }^{1,2}$, Neil Sinclair ${ }^{1,3}$, Mian Zhang ${ }^{1,2}$, Nathalie Picqué ${ }^{4}$, \\ and Marko Loncar ${ }^{1}$ \\ ${ }^{1}$ John A. Paulson School of Engineering and Applied Sciences, Harvard University, Cambridge, Massachusetts 02138, USA \\ ${ }^{2}$ HyperLight Corporation, 501 Massachusetts Ave, Cambridge, MA 02139, USA \\ ${ }^{3}$ Division of Physics, Mathematics and Astronomy, and Alliance for Quantum Technologies (AQT), California Institute of Technology, 1200 E. \\ California Boulevard, Pasadena, CA 91125, USA \\ ${ }^{4}$ Max-Planck Institut für Quantenoptik, Hans Kopfermann Strasse 1, 85748 Garching, Germany
}

Authore-mail address: ashamsansari@seas.harvard.edu

\begin{abstract}
We demonstrate a low-repetition-rate lithium niobate based electro-optic (EO) frequency comb operating with $3.481 \mathrm{GHz}$ line spacing, by integrating a cavity-based EO comb source with traveling-wave phase-modulators on the same chip. $\odot 2020$ The Author(s)
\end{abstract}

On-chip integrated optical frequency comb sources have found numerous applications in science and technology, including frequency metrology, realization of optical clocks, spectroscopy, ranging, and microwave photonics. For many applications, including spectroscopy [1] and ranging [2], it is advantageous to employ a comb with low repetition rate which enhances the resolution of the measurement. For example, to achieve a doppler-limited resolution in dualcomb spectroscopy in the gas-phase, it is essential to have small comb line spacing [1]. This is challenging for most on-chip frequency comb sources, typically realized using third-order nonlinearity (i.e. $\chi^{(3)}$ ) and dispersion engineering of the material [3]. Such Kerr-combs featuring GHz-repetition-rates have been demonstrated recently in $\mathrm{SiN}$ and $\mathrm{SiO}_{2}$ platform $[4,5]$. An alternative approach to generate a frequency comb is based on the $\chi^{(2)}$ nonlinearity (Pockels effect) of the material. Recently, lithium-niobate on insulator (LNOI) has emerged as a promising platform to realize such electro-optic (EO) frequency combs [6,7]. Here, microwave-frequency modulation of optical fields in ultra-low loss integrated lithium niobate optical cavities results in cascaded sideband generation yielding broadband and phasecoherent spectra down to $10 \mathrm{GHz}$ spectral mode spacings [6]. In order to further reduce the repetition rate to $1-\mathrm{GHz}$ range larger resonators are needed, which may compromise the footprint, performance, electrical power consumption, and the comb bandwidth of the device. An alternative approach to address this issue is to fill-up the comb spacing $\left(f_{\text {rep }}\right)$ by an integer factor $(m)$ through electro-optic phase modulation, by driving the phase modulator (PM) at frequency $f_{P M}=f_{\text {rep }} / m$ (Fig. 1(a)).
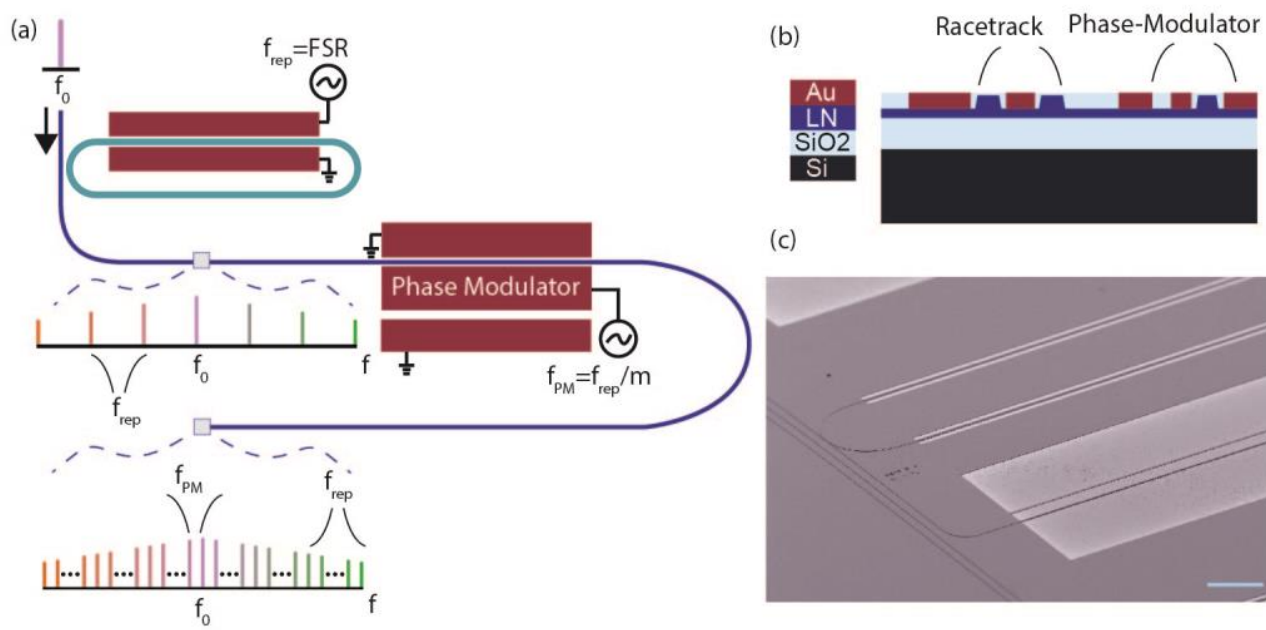

(c)

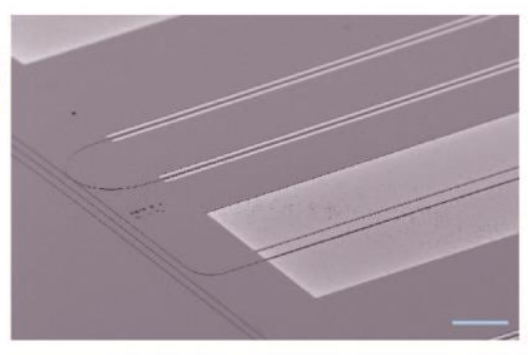

Fig. 1. (a) General scheme of the electro-optic combs sources with tunable FSR on-chip (a) A low-loss cavity-based modulator is driven with a microwave frequency of $f_{\text {rep }}$ equal to its FSR. The output of the comb passes through an integrated electro-optic phase modulator that fills the spectral gaps between the comb lines by a factor of $m$. (b) Cross-section schematic of our fabricated structure. (c) Scanning electron micrograph of a portion of the fabricated device, showing parts of the ring and phase modulator. The scale-bar represents 100- m. 
Here we use this approach to demonstrate an EO-comb with the repetition rate of $f_{\text {rep }}=3.481 \mathrm{GHz}$. First, a cavity based EO comb with $10.453 \mathrm{GHz}$ repetition rate is realized using the LNOI platform [6]. Next, the generated comb signal is passed through a travelling wave phase modulator [8], fabricated on the same chip, driven by only a sinusoidal signal of frequency $f_{P M}=10.453 / 3=3.481 \mathrm{GHz}$ (Fig 1(a) and (b)). In this device, the phase modulators RF signal is coherently locked to the RF signal driving the ring resonator. The phase modulation yields an increase in the density of the states in the frequency domain by a factor of $m=3$ (Fig.2), resulting in the output comb spectrum with a decreased FSR of 3.481 GHz. This approach potentially allows for a wide range of tunable FSRs since the phase-modulator can support low-noise modulation at frequencies in the MHz range. Furthermore, it has been shown that the comb can be filled with any integer fraction of the initial FSR, if more complex waveforms are used for phase modulation [9]. This will be subject to future work.

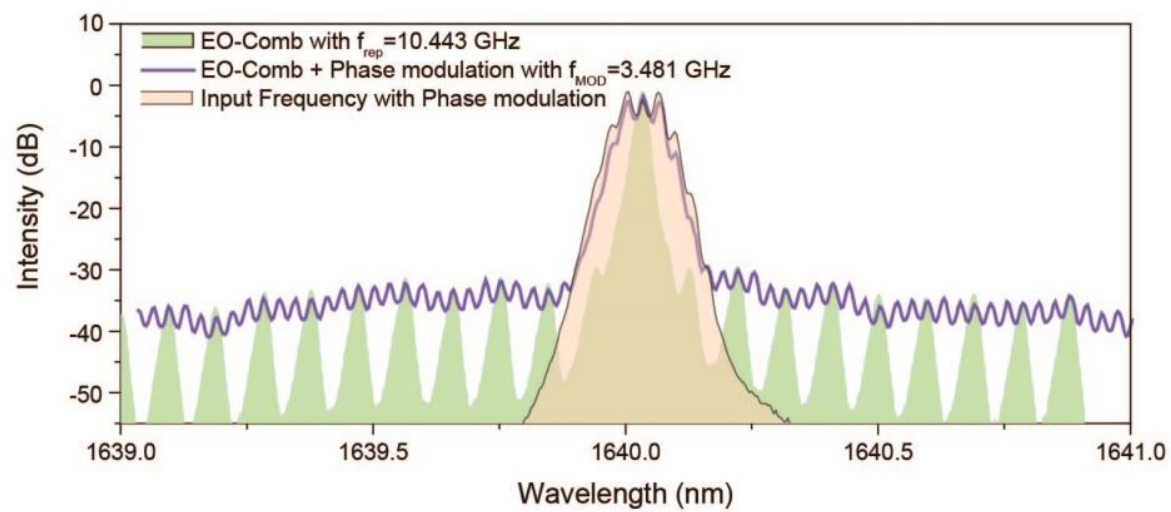

Fig. 2. The measured output spectrum of the low-repetition-rate EO-comb, including the original-comb with FSR $=10.443 \mathrm{GHz}$ and the phase-modulated comb with FSR=3.481 GHz. (The extinction of each line is limited by the optical spectrum analyzer maximum resolution.)

In conclusion, we demonstrated an integrated EO-comb on the LNOI platform by cascading a cavity-circulated and a pass-through phase-modulator with a low-repetition-rate $(\mathrm{FSR}=3.481 \mathrm{GHz})$, for the first time. This platform could potentially generate combs in the $\mathrm{MHz}$ range with $\mathrm{THz}$ spanning spectrum.

\section{Acknowledgments}

This work is supported by Air Force Office of Scientific Research (AFOSR Award number: FA9550-19-1-0310). Device fabrication was performed at the Center for Nanoscale Systems (CNS) at Harvard University.

\section{References}

1. N. Picqué, and T. W. Hänsch, "Frequency comb spectroscopy," Nature Photonics 13, 146-157 (2019).

2. P. Trocha, M. Karpov, D. Ganin, M. H. P. Pfeiffer, A. Kordts, S. Wolf, J. Krockenberger, P. Marin-Palomo, C. Weimann, S. Randel, W. Freude, T. J. Kippenberg, and C. Koos, "Ultrafast optical ranging using microresonator soliton frequency combs," Science 359, 887-891 (2018).

3. A. L. Gaeta, M. Lipson, and T. J. Kippenberg, "Photonic-chip-based frequency combs," nature photonics 13, 158-169 (2019).

4. M.-G. Suh, and K. Vahala, "Gigahertz-repetition-rate soliton microcombs," Optica 5, 65-66 (2018).

5. B. Shen, L. Chang, J. Liu, H. Wang, Q.-F. Yang, C. Xiang, R. N. Wang, J. He, T. Liu, and W. Xie, "Integrated turnkey soliton microcombs operated at CMOS frequencies," arXiv preprint arXiv:1911.02636 (2019).

6. M. Zhang, B. Buscaino, C. Wang, A. Shams-Ansari, C. Reimer, R. Zhu, J. M. Kahn, and M. Lončar, "Broadband electro-optic frequency comb generation in a lithium niobate microring resonator," Nature 568, 373 (2019).

7. M. Zhang, C. Wang, R. Cheng, A. Shams-Ansari, and M. Lončar, "Monolithic ultra-high-Q lithium niobate microring resonator," Optica 4, 1536-1537 (2017).

8. T. Ren, M. Zhang, C. Wang, L. Shao, C. Reimer, Y. Zhang, O. King, R. Esman, T. Cullen, and M. Lončar, "An integrated lowvoltage broadband lithium niobate phase modulator," IEEE Photonics Technology Letters 31, 889-892 (2019).

9. A. Malacarne, and J. Azaña, "Discretely tunable comb spacing of a frequency comb by multilevel phase modulation of a periodic pulse train," Opt. Express 21, 4139-4144 (2013) 